\title{
PREPRINTS
}

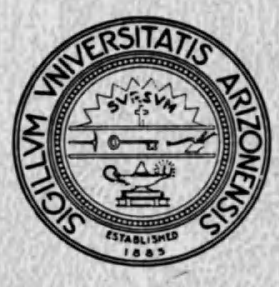

\author{
OF THE \\ STEWARD \\ OBSERVATORY \\ THE UNIVERSITY OF ARIZONA \\ TUCSON, ARIZONA
}

PULSATION CONSTANTS AND DENSITIES FOR DOUBLE

MODE VARIABLES IN THE CEPHEID INSTABILITY STRIP

W. S. FITCH

Steward Observatory, University of Arizona

Submitted for Publication to The Astrophysical Journal 


\section{PULSATION CONSTANTS AND DENSITIES FOR DOUBLE \\ MODE VARIABLES IN THE CEPHEID INSTABILITY STRIP}

by

W. S. FITCH

Steward Observatory, University of Arizona

Submitted for Publication to The Astrophysical Journal 


\section{A BST'RACT}

Pulsation constants and period ratios are given for the first six radial pulsation modes of a set of seven polytropes with indices ranging from $n=2.25$ to $n=4.00$. The polytrope results are combined with published theoretical data from stellar mode1 pulsation calculations and observational data on thirteen double mode variables in the Cepheid instability strip to obtain four interpolation formulae relating fundamental periods $\mathrm{P}_{\mathrm{O}}$, pulsation constants $Q_{0}$, and period ratios $P_{1} / P_{0}$ and $P_{2} / P_{1}$. The derived relations are used to obtain accurate densities for the thirteen observed variables, which include six dwarf Cepheids or AI Vel stars, one $\delta$ Sct star, one RR Lyr star, and five Cepheids. Approximate masses and luminosities are obtained for some of these stars. The probable connection between mass-luminosity ratios and the existence of double mode variables is discussed. 


\section{INTRODUCTION}

The existence of Cepheid strip variables with two simultaneously excited radial pulsation modes has been known for some time, and there are at present at least twelve such stars whose periods have been unambiguously determined. The first extensive theoretical calculations relating to these stars are those of Schwarzchild (1941), while the theoretical and observational aspects of the problem were thoroughly reviewed by Ledoux and Walraven (1958). Most recently, Christy (1966a) has given a comprehensive and very illuminating discussion of these stars, but his treatment can be improved on the observational side by some data revisions and additions and on the theoretical side by the inclusion of higher overtone eigenvalues.

Accordingly, to augment results from accurate ste1lar mode1 calculations, we here present ( $\S$ II) an extension of Schwarzchild's (1941) standard model computations to the first six radial pulsation modes of a set of seven polytropes with indices ranging from $\mathrm{n}=2.25$ to $\mathrm{n}=4.00$. In $\S$ III we summarize the observational data available at present and obtain accurate pulsation constants and mean densities for thirteen observed double mode variables with periods in the range 0.05 to 3 days; and in $\S$ IV we discuss the masses and luminosities of some of these stars, and the relation between mass-luminosity ratios and the existence of double-mode variables. 
II. PULSATION CONSTANTS AND PERIOD RATIOS FOR POLYTROPES

For each polytropic index $\mathrm{n}$, the Lane-Emden equation (cf. Chandrasekhar 1939) was integrated outward to determine the polytropic radius $\xi_{1}$ and the density ratio $\rho c /\langle\rho\rangle$, and the resulting values, displayed in Table 1, agree satisfactorily with those listed by Chandrasekhar (1939, Table 4). The tabular values obtained for the Lane-Emden variable $\theta$ and its first derivative were used to evaluate the coefficients in the linear adiabatic wave equation for radial pulsations (Schwarzchild 1941, eq. [2]; Rosseland 1949, eq. [3.32]), and, following Schwarzchild, the wave equation was then integrated from center out and from surface in, with most eigenvalues determined by fitting the two integrations near $0.7 \xi_{1}$. In a few cases, convergence to the desired mode could not be obtained until the fitting point was moved to $0.8 \xi_{1}$. To cast the results into the most useful form, we adopt $\mathrm{G}=6.670 \times 10^{-8} \mathrm{gm}^{-1} \mathrm{~cm}^{3} \mathrm{sec}^{-2}$ and $\left\langle\rho_{\odot}\right\rangle=1.409 \mathrm{gm} \mathrm{cm}^{-3}$, and express the eigenvalues (Rosseland 1949, eq. [3.35]) as pulsation constants $\left.Q_{k}=P_{k} \sqrt{ }\left(\langle\rho\rangle \quad /<\rho_{0}\right\rangle\right)$, where $\langle\rho>$ is the mean density of the polytrope and $\mathrm{P}_{\mathrm{k}}$ is the pulsation period (in days) of the $\mathrm{k}^{\text {th }}$ radial mode (i.e., $k$ is the number of pulsation amplitude nodes between center and surface, so that $P_{0}$ is the fundamental period). In Table 2 we present 161 pulsation constants arranged as functions of the polytropic index $n$, the mode number $k$, and the ratio of specific heats $T$, expressed by the parameter $\alpha=3-4 / T$. For convenience, we also give the ratio of successive periods $\mathrm{P}_{\mathrm{k}+1} / \mathrm{P}_{\mathrm{k}}$. 
Al1 integrations were performed with a single-precision Runge-Kutta integration routine, and, to simplify programing, a fixed step-length in $\xi$ was used $(\Delta \xi=0.02$ for the polytrope integrations, except $\Delta \xi=0.015$ at $n=2.25$ ), with the wave equation step-length twice that for the polytrope equation.

A few test integrations with smaller step-length indicate that the computational accuracy of $Q_{k}$ is about $10^{-6}$ day for most of the fundamental modes, but falls off rapidly with increasing order to about $10^{-4}$ day at $k=5$. Similarly, the period ratios are accurate to one or two units in the last place at $(n=3.0$; $k=0,1$ ), but may be in error by as much as 50 units at $k=4,5$. Therefore, the pulsation constants and period ratios for the higher modes should be used only for indicating the general trends with $\mathrm{n}, \mathrm{k}$, and $\mathrm{T}$. We obtain good agreement with Schwarzchild's (1941) values for $\mathrm{n}=3$.

For convenience in the following discussion, for the polytropes with $\mathrm{T}=5 / 3(\alpha=0.6)$ we have fitted $\mathrm{P}_{1} / \mathrm{P}_{0}$ and $\mathrm{P}_{2} / \mathrm{P}_{1}$ as power series functions of each other and obtained

$$
\begin{aligned}
& \mathrm{P}_{1} / \mathrm{P}_{\mathrm{O}}=0.7327+1.70\left(\mathrm{P}_{2} / \mathrm{P}_{1}-0.77\right)-19.8\left(\mathrm{P}_{2} / \mathrm{P}_{1}-0.77\right)^{2}+72\left(\mathrm{P}_{2} / \mathrm{P}_{1}-0.77\right)^{3} \\
& \pm 0.0017 \pm 0.09 \quad \pm_{1.1} \quad \pm 42 \text { m.e. }
\end{aligned}
$$

and

$$
\begin{aligned}
\mathrm{P}_{2} / \mathrm{P}_{1} & =0.7511+0.386\left(\mathrm{P}_{1} / \mathrm{P}_{\mathrm{o}}-0.7\right)+3.89\left(\mathrm{P}_{1} / \mathrm{P}_{\mathrm{o}}-0.7\right)^{2}+28\left(\mathrm{P}_{1} / \mathrm{P}_{\mathrm{o}}-0.7\right)^{3}, \\
& \pm 0.0026 \pm 0.067 \quad \pm_{0.57}
\end{aligned}
$$

with errors not exceeding 0.003 and 0.004 , respectively, in the range of values shown in Table 2 . 
III. STARS WITH TWO EXCITED MODES

As discussed by Ledoux and Walraven (1958) and by Christy (1966a), the period ratio $\mathrm{P}_{1} / \mathrm{P}_{0}$ is theoretically a fairly well defined function of the fundamental pulsation constant $Q_{0}$, and in Table 3 we have assembled results of stellar model calculations by a number of authors. The sources chosen were an evolutionary sequence of models of a $7 \mathrm{M}_{\theta}$ star by Baker and Kippenhahn (1965); six models by Dumezil-Curien, by Epstein, and by Keller, as 1isted by Ledoux and Walraven (1958, Table 12); a $6.3 \mathrm{M}_{\ominus}$ model of $n$ Aq1 by Christy (1966c); four RR Lyr star transition-zone models by Christy (1966b); and the $T=5 / 3$ polytrope calculations from Table 2 of this paper. As seen in Figure 1, these 25 models agree we11 with each other but display a significant scatter, so all points were treated as of equal weight in deriving the theoretical dependence of $Q_{0}$ (days) on $\mathrm{P}_{1} / \mathrm{P}_{0}$ in the form:

$$
\begin{aligned}
& Q_{0}=0.0479-0.0952\left(P_{1} / P_{0}-0.65\right)-0.225\left(P_{1} / P_{0}-0.65\right)^{2} \text {. } \\
& \pm 0.0005 \pm 0.0047 \quad \pm 0.057 \text { m.e. }
\end{aligned}
$$

The maximum residual of the Table 3 data from this expression is 0.0031 day, and the mean error of a single measure is only 0.0014 day. We emphasize that equation (3) is not suitable for extrapolating outside the range of values considered.

In Table 4 we present the pertinent data for thirteen Cepheid strip variable stars with two excited radial pulsation modes. Of these stars, which include six dwarf Cepheids or AI Vel stars, one $\delta$ Sct star, one RR Lyr star, and five Cepheids, all but the $\delta$ Sct star 4 CVn have very well defined and unambiguous double periodicities. 
The observational data for the latter is fragmentary, and further measurements are desirable, but the periods reported by Smyth and Melville (1967) and repeated here are probably correct. We note that of the nine stars 1isted by Christy (1966a, Tables 1 and 2) in his discussion of this group, $\delta$ Sct (Fitch 1960; Leung and Wehlau 1967) and DQ Cep (Fitch and Wehlau 1965) should not be included, and that the period ratio adopted by Christy for VZ Cnc is incorrect. Examination of Figure 2 of Fitch (1955) shows that in VZ Cnc, the secondary period is shorter than the primary, and the periods given there are correct. (This point is independently established by the excellent analytic representation of the photometry possible through a doubly harmonic expansion in the two published periods.) Columns 3 and 2 of Table 4 give the observed period ratio and longer period, respectively. It is of interest to note here that in V703 Sco the shorter period has the largest amplitude, and that in AI Ve1 and VX Hya the two amplitudes are nearly equal, while in the remaining stars the longer period is of greatest amplitude.

We illustrate these observational results in Figure 2, where the very precise correlation of $\mathrm{P}_{1} / \mathrm{P}_{0}$ with $\log \mathrm{P}_{0}$ (days) is clearly seen. Since VZ Cnc alone does not fit this correlation, we have omitted it from the group in deriving the empirical interpolation formula

$$
\begin{aligned}
& \mathrm{P}_{1} / \mathrm{P}_{\mathrm{o}}=0.7291-0.0603 \log \mathrm{P}_{\mathrm{o}}+0.008\left(\log \mathrm{P}_{\mathrm{o}}\right)^{2}+0.021\left(\log \mathrm{P}_{\mathrm{o}}\right)^{3} \\
& \pm 0.0031 \pm 0.0037 \quad \pm 0.013 \\
& \pm 0.010 \mathrm{~m} . \mathrm{e} .
\end{aligned}
$$

The mean error of a single measure as determined by residuals from this expression is 0.0041 , while the maximum residual is 0.0074 , and the 
relation (4) is shown graphically as the lower curve in Figure 2. We also show here the corresponding data for the transition-zone RR Lyr star models by Christy (1966b), the $n$ Aq1 mode1 by Christy (1966c), and the $7 \mathrm{M}_{\theta}$ Cepheid models by Baker and Kippenhahn (1965), used previously in Table 3 and Figure 1. Christy's (1966b) investigation of the instability phenomena in RR Lyr stars would lead us to expect that any real star exhibiting two excited pulsation modes must be at an evolutionary stage very near the transition between these two modes, and the excellent agreement shown in Figure 2 between the empirical equation (4) relation and Christy's theoretical results for the fundamental-first overtone mode transition confirms our fundamental mode designation in column 4 of Table 4 for these twelve stars.

The $T=5 / 3$ polytrope eigenvalues for the two lowest modes agree so well with the stellar model results (cf. Fig. 1) that we expect the polytrope third mode calculations will also agree, when corresponding model results become available. Therefore, we feel justified in combining the empirical relation (4) with the polytrope interpolation equation (2) to obtain the semi-empirical dependence of $\mathrm{P}_{2} / \mathrm{P}_{1}$ on $\log$ $\mathrm{P}_{\mathrm{O}}$, as shown by the upper curve in Figure 2 . The point representing VZ Cnc appears to be associated with this line, so we identify the observed periods in VZ Cnc as the first and second overtones, and employ equation (1) to obtain $\mathrm{P}_{1} / \mathrm{P}_{0}=0.7682$, which implies that the (unexcited) fundamental period is 0.2322 day. The point representing this inferred $\mathrm{P}_{\mathrm{O}}$ and the observed $\mathrm{P}_{2} / \mathrm{P}_{1}$ is plotted as an open circle in Figure 2 and is seen to lie on top of the semi-empirical relation. Similarly, if we 
adopt this inferred period $\mathrm{P}_{\mathrm{O}}$, then equations (4) and (2) lead to a calculated value of $\mathrm{P}_{2} / \mathrm{P}_{1}=0.8006$, the same as was observed. While this precise agreement is probably fortuitous, we conclude that VZ Cnc is in fact a first and second overtone mode pulsator.

With the established $\mathrm{P}_{1} / \mathrm{P}_{\mathrm{o}}$ ratios, we now use equation (3) to yield the fundamental period pulsation constants shown in column 6 of Table 4 and then obtain the corresponding densities listed in column 7 . It is difficult to assess the accuracy of these results, but the internal consistency of both the observational and the theoretical data suggests that the pulsation constants are not likely to be in error by more than about two percent. Since the errors in the periods are negligible, the corresponding mean densities should be good to about five percent.

The apparent discontinuity between the $\mathrm{P}_{1} / \mathrm{P}_{0}$ data on the right-and left-hand sides of Figure 2 requires comment. From our previous discussion, the data on the left, both observational and theoretical, refer to stars at the transition between pulsation modes, whereas the evolutionary sequence at the right is for a $7 \mathrm{M}_{\ominus}$ star traversing the Cepheid strip from right to left on its second post-main-sequence crossing, and the linear, nonadiabatic calculations employed could not show clearly which pulsation mode would be dominant. Christy's (1966c) nonlinear computations on a $6.3 \mathrm{M}_{\theta}$ model representing $\eta$ Aql agree $\wedge$ eirly wel1 with Baker and Kippenhahn's (1965) $7 \mathrm{M}_{\odot}$ sequence so far as periods and pulsation constants are concerned, but indicate that the fundamental mode would be unexcited at about model 12 of their sequence. How much earlier in the evolutionary sequence the transition from fundamental to first overtone would take place is not presently clear, but there does not seem to be any necessary contradiction between the smooth empirical 
relation on the left-hand side of Figure 2 and the theoretical sequence on the right.

$$
\text { IV. DISCUSSION }
$$

The most immediate applications of the preceding results concern the masses and luminosities of these stars.

\section{a) Masses}

Designating the relative errors in mass $M$, radius $R$, mean density $\left\langle\rho>\right.$, and surface gravity $g$ by $\varepsilon_{M}=\Delta M / M, \varepsilon_{R}=\Delta R / R$, $\varepsilon_{\rho}=\Delta\langle\rho\rangle /\langle\rho\rangle$, and $\varepsilon_{g}=\Delta \mathrm{g} / \mathrm{g}$, respectively, we have $\varepsilon_{M}^{2}=\varepsilon_{\rho}^{2}+9 \varepsilon_{R}^{2}=4 \varepsilon_{\rho}^{2}+9 \varepsilon_{g}^{2}$. Adopting our estimated upper limit on $\varepsilon_{\rho} \leqslant 0.05$ (§ III), it follows that a mass determination good to 25 percent will require a radius measurement with $\varepsilon_{R} \leqslant 0.08$ or a surface gravity measurement with $\varepsilon_{g} \leqslant 0.08$. Therefore, significant information concerning the evolutionary state of these stars from considerations of mass loss can only be obtained for those stars with very precisely known radii or gravities.

Apparently, accurate radii via some form of the Wesselink method are not yet available for any of the Table 4 stars. From spectrophotometric investigations, Besse11 (1969) has given log $\mathrm{g}$ for SX Phe, $\mathrm{AI} V \mathrm{Ve}$, and $\mathrm{VZ} \mathrm{Cnc}$ as $4.0 \pm 0.1,3.5 \pm 0.1$, and $3.1 \pm 0.2$, respectively. Since

$$
\log \left(M / M_{0}\right)=3 \log g-2 \log (<\rho>/<\rho \theta)-13.314,
$$

we find that the corresponding masses are $\mathrm{M} / \mathrm{M}_{\theta}=0.43,0.21$, and 0.22 , with minimum uncertainties of factors 2,2 , and 4, respectively. These 
masses are al1 somewhat smaller than the values adopted by Besse11, and if taken at face value they do substantiate the mass loss which he suggested for the AI Vel stars. However, the errors, which here depend almost entirely on the gravity measures, are too large to definitely preclude the possibility of post-main-sequence evolution with no mass loss. Thus the AI Vel stars may be on one of the first crossings of the instability strip, not having gone through a red giant phase yet.

\section{b) Luminosities}

In his studies of RR Lyr and Cepheid models, Christy (1966b) found that the growth or decay rate for the pulsation amplitude at a given period (or luminosity) depended on the mass. Large mass stars have much larger pulsational kinetic energies and therefore change amplitude much slower than do low mass stars. Models representing typical cluster variables generally had relatively low mass envelopes and rapid amplitude change rates when not pulsating at maximum amplitude. In following such a star across the instability strip, either the fundamental or first overtone was initially excited (depending on whether the direction of evolution was toward higher or lower $\mathrm{T}_{e}$, respectively), and when a transition to the other mode occurred, it ordinarily was complete in about 100 periods. Therefore a normal cluster variable is very unlikely to be observed in the act of transition. 
Since the rapidity of mode transition depends on the amplitude change rate and ultimately on the luminosity-to-mass ratio, one concludes that the observed double mode variables have low $\left(L / L_{0}\right) /$ $\left(M / M_{\ominus}\right)$, relative to a typical $R R$ Lyr star. Our identification of VZ Cnc as a first and second overtone pulsator with unexcited fundamenta1, and of V703 Sco as having larger amplitudek in first overtone than in fundamental, when viewed together with Christy's (1966b) finding that at very low luminosity-to-mass ratio the instability strip is entirely occupied by first overtone pulsators, helps strengthen this conclusion. Further evidence in favor of a low ratio comes from the Detres' (1966) observation that the dwarf Cepheids show much more stable periods than do the cluster variables, thus implying an envelope structure much less sensitive to sma11 perturbations.

In addition, we expect that any observed double mode star (fundamental and first overtone) must be in or very near the transitionzone gap characteristic of cluster variables (Christy 1966b), and that it will have a present fundamental period $\mathrm{P}_{\mathrm{o}}$ nearly the same as the transition period $\mathrm{P}_{\operatorname{tr}}$ which Christy defined. Were this not so, a much larger scatter should be observed in the $P_{1} / P_{0}$ vs. $\log P_{0}$ curve of Figure 2. For stars at this transition, Christy found a periodluminosity relation which we express here as

$$
\mathrm{M}_{\mathrm{bo} 1}=-0.50-4.17 \log \mathrm{P}_{\mathrm{tr}} \text { (days), }
$$

where we have adopted +4.69 as the bolometric magnitude of the sun (Harris 1963). Christy (1968) indicated that this relation is valid in 
the range $+2.2>\mathrm{M}_{\mathrm{bol}} \gtrsim-6.1$, and we have employed it to obtain the magnitudes given in the last column of Table 4. The application of this expression to $\mathrm{VZ} \mathrm{Cnc}$ is questionable, since the evolutionary change necessary to take it to $\mathrm{P}_{\operatorname{tr}}$ (fundamental) should be accompanied by a decrease in $\langle\rho\rangle$ from its present value. Probably $P_{\text {tr }}$ is greater than the present unexcited $P_{0}$, since $Q_{0}$ is nearly constant for the shorter period stars (cf. Table 4). If evolution proceeds across the instability strip at constant luminosity, we have underestimated the brightness of VZ Cnc.

Accurate radius determinations for all of these stars are urgently needed to determine their masses and help resolve the questions relating to their evolutionary states and mass losses.

I am greatly indebted to Dr. Thomas E. Margrave for his continued interest in this problem, and for finding and correcting an error in the original polytrope eigenvalue program. I wish to thank Dr. Ray J. Weymann for providing a copy of his Runge-Kutta integration routine and for his advice on fitting procedures for the pulsation integrations. The calculations were carried out on the CDC 6400 at the University of Arizona Computer Center, and I am grateful to the personnel there for their assistance. 
TABIE 1

\begin{tabular}{l|r|r}
\multicolumn{3}{c}{ POLYTROPIC CONSTANTS } \\
\hline $\mathrm{n}$ & \multicolumn{1}{c|}{$\xi 1$} & $\rho \mathrm{c} /\langle\rho\rangle$ \\
\hline 2.25 & 4.80551 & 16.1459 \\
2.50 & 5.35528 & 23.4067 \\
2.75 & 6.03558 & 34.9513 \\
3.00 & 6.89685 & 54.1835 \\
3.25 & 8.01895 & 88.1549 \\
3.50 & 9.53585 & 152.887 \\
4.00 & 14.97164 & 622.422
\end{tabular}

TABLE 4

CEPHE ID STRIP VARIABLES WITH TWO EXCITED MODES

\begin{tabular}{r|c|c|c|c|c|c|c}
\hline STAR & $\mathrm{P}_{k}$ (days) & $\mathrm{P}_{k+1} / \mathrm{P}_{\mathrm{k}}$ & $\mathrm{k}$ & $\log \mathrm{P}_{\mathrm{o}}$ & $\mathrm{Q}_{\mathrm{o}}$ (days) & $\langle\rho\rangle /\left\langle\rho_{0}\right\rangle$ & $\mathrm{M}_{\mathrm{bol}}$ \\
\hline SX Phe & 0.05496 & 0.7782 & 0 & -1.2600 & 0.0320 & $3.39 \times 10^{-1}$ & \\
RV Ari & 0.09313 & .7726 & 0 & -1.0309 & .0328 & $1.24 \times 10^{-1}$ & \\
AI Ve1 & 0.11157 & .7727 & 0 & -0.9525 & .0328 & $8.64 \times 10^{-2}$ & \\
V703 Sco & 0.14996 & .7683 & 0 & -0.8240 & .0335 & $4.99 \times 10^{-2}$ & \\
4 CVn & 0.16553 & .7722 & 0 & -0.7811 & .0329 & $3.95 \times 10^{-2}$ & +2.2 \\
VZ Cnc & 0.17836 & .8006 & 1 & -0.6343 & .0335 & $2.08 \times 10^{-2}$ & +2.2 \\
VX Hya & 0.22339 & .7732 & 0 & -0.6509 & .0328 & $2.16 \times 10^{-3}$ & +2.2 \\
AC And & 0.71124 & .7383 & 0 & -0.1480 & .0377 & $2.81 \times 10^{-4}$ & +0.1 \\
V439 Oph & 1.89296 & .709 & 0 & +0.2771 & .0415 & $4.81 \times 10^{-4}$ & -1.7 \\
TU Cas & 2.13930 & .7097 & 0 & +0.3303 & .0414 & $3.75 \times 10^{-4}$ & -1.9 \\
U TrA & 2.56844 & .7105 & 0 & +0.4097 & .0413 & $2.59 \times 10^{-4}$ & -2.2 \\
AP Ve1 & 3.12781 & .7031 & 0 & +0.4952 & .0422 & $1.82 \times 10^{-4}$ & -2.6 \\
BK Cen & 3.1740 & 0.7047 & 0 & +0.5016 & 0.0420 & $1.75 \times 10^{-2}$ & -2.6 \\
\hline
\end{tabular}

* SX Phe: (Walraven 1955a); RV Ari: (Detre 1956); AI Ve1: (Walraven 1955b); V703 Sco: (Oosterhoff 1966X); 4 CVn: (Smyth and Melville 1967); VZ Cnc: (Guman 1955; Fitch 1955); VX Hya: (Fitch 1966); AC And: (Lange 1963); V439 Oph: (Gusev 1967); TU Cas: (Oosterhoff 1957); U TrA: (Jansen 1962); AP Ve1: (Oosterhoff 1964); BK Cen: (Leotta - Janin 1967). 
TABLE 2

POLYTROPE PULSATION CONSTANTS AND PERIOD RATIOS

\begin{tabular}{|c|c|c|c|c|c|c|c|c|c|}
\hline \multirow{2}{*}{$\mathrm{n}$} & \multirow{2}{*}{$\mathrm{k}$} & \multicolumn{4}{|c|}{$\mathrm{Q}_{\mathrm{k}}$ (days) } & \multicolumn{4}{|c|}{$\mathrm{P}_{\mathrm{k}+1} / \mathrm{P}_{\mathrm{k}}$} \\
\hline & & $\alpha=0.0$ & 0.2 & 0.4 & 0.6 & 0.0 & 0.2 & 0.4 & 0.6 \\
\hline \multirow[t]{6}{*}{2.25} & 0 & & 0.094159 & 0.065334 & 0.052230 & & 0.3901 & 0.5171 & 0.5949 \\
\hline & 1 & 0.039971 & .036729 & .033781 & .031071 & 0.6719 & .6896 & .7060 & .7211 \\
\hline & 2 & .026856 & .025330 & .023849 & .022406 & .7653 & .7725 & .7794 & .7860 \\
\hline & 3 & .020554 & .019568 & .018588 & .017610 & .8151 & .8189 & .8226 & .8261 \\
\hline & 4 & .016753 & .016024 & .015290 & .014547 & .8469 & .8492 & .8513 & .8536 \\
\hline & 5 & .014188 & .013607 & .013017 & .012417 & & & & \\
\hline \multirow[t]{6}{*}{2.50} & 0 & & .083098 & .058163 & .046940 & & .4355 & .5691 & .6452 \\
\hline & 1 & .039597 & .036188 & .033103 & .030284 & .6807 & .7002 & .7184 & .7353 \\
\hline & 2 & .026954 & .025339 & .023780 & .022269 & .7696 & .7777 & .7854 & .7928 \\
\hline & 3 & .020744 & .019705 & .018676 & .017655 & .8177 & .8220 & .8262 & .8302 \\
\hline & 4 & .016962 & .016197 & .015430 & .014658 & .8485 & .8511 & .8537 & .8561 \\
\hline & 5 & .014392 & .013786 & .013172 & .012549 & & & & \\
\hline \multirow[t]{6}{*}{2.75} & 0 & & .072600 & .051500 & .042177 & & .4896 & .6269 & .6953 \\
\hline & 1 & .039143 & .035547 & .032285 & .029324 & .6902 & .7116 & .7324 & .7520 \\
\hline & 2 & .027015 & .025295 & .023645 & .022052 & .7739 & .7830 & .7919 & .8005 \\
\hline & 3 & .020908 & .019807 & .018724 & .017652 & .8202 & .8251 & .8298 & .8346 \\
\hline & 4 & .017149 & .016343 & .015538 & .014732 & .8503 & .8533 & .8564 & .8592 \\
\hline & 5 & .014582 & .013946 & .013306 & .012657 & & & & \\
\hline \multirow[t]{6}{*}{3.00} & 0 & & .062730 & .045476 & .038100 & & .5546 & .6875 & .7382 \\
\hline & 1 & .038616 & .034787 & .031264 & .028125 & .7003 & .7244 & .7492 & .7723 \\
\hline & 2 & .027044 & .025198 & .023424 & .021722 & .7789 & .7894 & .7999 & .8103 \\
\hline & 3 & .021065 & 019891 & .018737 & .017601 & .8237 & .8294 & .8351 & .8409 \\
\hline & 4 & .017351 & .016498 & .015648 & .014800 & .8541 & .8577 & .8612 & .8647 \\
\hline & 5 & .014820 & .014150 & .013476 & .012798 & & & & \\
\hline \multirow[t]{6}{*}{3.25} & 0 & & .053612 & .040337 & .034894 & & .6314 & .7416 & .7642 \\
\hline & 1 & .038017 & .033852 & .029915 & .026666 & .7111 & .7391 & .7710 & .7954 \\
\hline & 2 & .027033 & .025020 & .023065 & .021210 & .7839 & .7962 & .8094 & .8224 \\
\hline & 3 & .021191 & .019920 & .018669 & .017443 & .8261 & .8328 & .8399 & .8470 \\
\hline & 4 & .017505 & .016590 & .015681 & .014775 & .8551 & .8594 & .8637 & .8682 \\
\hline & 5 & .014968 & .014257 & .013543 & .012827 & & & & \\
\hline \multirow[t]{6}{*}{3.50} & 0 & & .045527 & .036436 & .032597 & & .7158 & .7723 & .7720 \\
\hline & 1 & .037352 & .032588 & .028139 & .025166 & .7225 & .7586 & .7983 & .8133 \\
\hline & 2 & .026985 & .024720 & .022464 & .020468 & .7893 & .8047 & .8230 & .8373 \\
\hline & 3 & .021299 & .019893 & .018488 & .017137 & .8291 & .8375 & .8471 & .8562 \\
\hline & 4 & .017660 & .016661 & .015662 & .014672 & .8570 & .8623 & .8682 & .8740 \\
\hline & 5 & .015135 & .014367 & .013597 & .012824 & & & & \\
\hline \multirow[t]{5}{*}{4.00} & 0 & & .035419 & .032192 & .029781 & & .7870 & .7763 & .7793 \\
\hline & 1 & .035839 & .027875 & .024992 & .023207 & .7467 & .8240 & .8201 & .8203 \\
\hline & $\begin{array}{l}2 \\
3\end{array}$ & $\begin{array}{l}.026761 \\
.021444\end{array}$ & $\begin{array}{l}.022970 \\
.019300\end{array}$ & $\begin{array}{l}.020495 \\
.017399\end{array}$ & $\begin{array}{l}.019037 \\
.016155\end{array}$ & $\begin{array}{l}.8013 \\
.8357\end{array}$ & $\begin{array}{l}.8402 \\
.8557\end{array}$ & $\begin{array}{l}.8489 \\
.8684\end{array}$ & $\begin{array}{l}.8486 \\
.8692\end{array}$ \\
\hline & 4 & .017921 & .016515 & .015109 & .014042 & 0.8598 & 0.8711 & 0.8821 & 0.8846 \\
\hline & 5 & 0.015408 & 0.014386 & 0.013328 & 0.012421 & & & & \\
\hline
\end{tabular}


TABLE 3

PERIOD RATIOS AND PULSATION CONSTANTS FROM STELIAR MODELS

\begin{tabular}{c|c|c||c|c|c||c|c|c}
\hline Mode1 & $\mathrm{P}_{1} / \mathrm{P}_{\mathrm{o}}$ & $\mathrm{Q}_{\mathrm{o}}$ (days) & Mode1 & $\mathrm{P}_{1} / \mathrm{P}_{\mathrm{o}}$ & $\mathrm{Q}_{\mathrm{o}}$ (days) & Mode1 & $\mathrm{P}_{1} / \mathrm{P}_{\mathrm{o}}$ & $\mathrm{Q}_{\mathrm{o}}$ (days) \\
\hline BK1 & 0.558 & 0.0553 & $\mathrm{n}=2.75$ & 0.695 & 0.0422 & $\mathrm{C} 13 \mathrm{c}, 13 \mathrm{e}$ & 0.748 & 0.0370 \\
LW7 & .543 & .0558 & $\mathrm{BK} 6$ & .712 & .0432 & $\mathrm{C} 15 \mathrm{~d}, 15 \mathrm{e}$ & .740 & .0371 \\
$\mathrm{n}=2.25$ & .595 & .0522 & $\mathrm{BK} 10$ & .731 & .0408 & $\mathrm{n}=3.25$ & .764 & .0349 \\
LW8 & .598 & .052 & $\mathrm{n}$ Aq1 & .735 & .0375 & $\mathrm{n}=3.50$ & .772 & .0326 \\
BK2 & .620 & .0496 & $\mathrm{n}=3.00$ & .738 & .0381 & $\mathrm{LW} 10$ & .795 & .0320 \\
$\mathrm{n}=2.50$ & .645 & .0469 & $\mathrm{BK} 15$ & .743 & .0389 & $\mathrm{LW} 11$ & .780 & .0314 \\
BK3 & .665 & .0468 & $\mathrm{C} 3 \mathrm{~b}, 3 \mathrm{~d}$ & .750 & .0361 & $\mathrm{LW12}$ & .765 & .0309 \\
BK4 & 0.679 & 0.0454 & $\mathrm{C} 9 \mathrm{e}, 9 \mathrm{f}$ & .743 & .0364 & $\mathrm{n}=4.00$ & .779 & .0298 \\
& & & & & & $\mathrm{LW} 13$ & 0.79 & 0.0292 \\
\hline
\end{tabular}

Notes to Table 3: $\quad B K=$ evolutionary sequence for a $7 \mathrm{M}_{0}$ Cephid, by Baker and Kippenhahn (1965); $L W=$ Models by Epstein, by Dumezil-Curien, and by Keller, as listed in Table 12 by Ledoux and Walraven (1958); C = transition-zone RR Lyrae models by Christy (1966b); $n=$ polytropes with $T=5 / 3$, from Table 2 of this paper; $n \quad$ Aql $=$ model of $n$ Aql by Christy $(1966 c)$. 


\section{REFERENCES}

Baker, N., and Kippenhahn, R. 1965, Ap.J., 142, 868.

Balazs-Detre, J., and Detre, L. 1966, Kleine Veroff. Remeis-

Sternw. Bamberg, No. 40, p. 184 .

Besse11, M.S. 1969, Ap. J. Supp1., 18, 195.

Chandrasekhar, S. 1939, An Introduction to the Study of Stellar

Structure (Chicago: University of Chicago Press), Ch.4.

Christy, R. F. 1966a, Ann. Rev. Astr. and Ap., 4, 353.

- $1966 \mathrm{~b}$, Ap.J. , 144, 108 .

- $1966 \mathrm{c}$, ibid. 145,340 .

- 1968, Quart.J.R.A.S., 9, 13.

Detre, L. 1956, Mitt. d. Sternw.Budapest-Szabadsaghegy, No. 40.

Fitch, W.S. 1955, Ap.J., 121, 690 .

. 1960 , ibid. $132,430$.

. 1966 , ibid. 143,852 .

Fitch, W. S., and Wehlau, W. 1965, Ap.J., $142,1616$.

Guman, I. 1955, Mitt, d. Sternw. Budapest-Szabadsaghegy, No. 36.

Gusev, E. B. 1967, I.A.U. Commission 27, Information Bu11., No. 186.

Harris, D. L. 1963, Basic Astronomical Data, ed. K.Aa. Strand

(Chicago: University of Chicago Press), p. 263.

Jansen, A. G. 1962, B.A.N., 16, 141.

Lange, G. A. 1963, Acad.Sci.U.S.S.R., Var.Star Bu11., 14, 352.

Ledoux, P., and Walraven, T. 1958, Hdb.d.Phys., ed.S.Flúgge

(Berlin: Springer-Verlag), 51, 353.

Leotta - Janin, C. 1967, B.A.N., 19, 169.

Leung, K.-C., and Wehlau, W. 1967, Ap.J., 149, 39.

Oosterhoff, P. Th. 1957, B.A.N., 13, 320 .

- 1964, ibid. 17,448 .

- 1966x, ibid. , 18, 140.

Rosseland, S. 1949, The Pulsation Theory of Variable Stars

(New York: Oxford University Press), Ch. 3.

Schwarzchild, M. 1941, Ap.J., 94, 245.

Smyth, M.J., and Melville, J.P. 1967, Observatory, 87, 237.

Walraven, Th. 1955a, B.A.N., 12, 57.

- 1955b, ibid., p. 223 . 
CAPTIONS

Fig.1 - Theoretical relation between fundamental mode pulsation constant $Q_{0}$ and the first overtone-tofundamental period ratio. Solid triangles: Evolutionary sequence for a $7 \mathrm{M}_{\odot}$ star by Baker and Kippenhahn; open triangle: $n$ Aq1 model by Christy; solid circles: miscellaneous models tabulated by Ledoux and Walraven; crosses: RR Lyr transitionzone models by Christy; squares: $T=5 / 3$ polytropes. Smooth curve is given by equation (3).

Fig. 2 - Observed and theoretical relations between radial mode period ratios and fundamental period. Solid circles: observed double-mode variables in the Cepheid strip; open circle: position of VZ Cnc if pulsating in first and second overtones; crosses: RR Lyr transitionzone models by Christy; solid triangles: $7 \mathrm{M}_{0}$ evolutionary sequence by Baker and Kippenhahn; open triangle: $n$ Aql model by Christy. The lower smooth curve is $\mathrm{P}_{1} / \mathrm{P}_{0}$ relation given by the empirical equation (4); upper smooth curve is semiempirical $\mathrm{P}_{2} / \mathrm{P}_{1}$ relation generated from equation (4) by means of equation (2). 
$-19-$
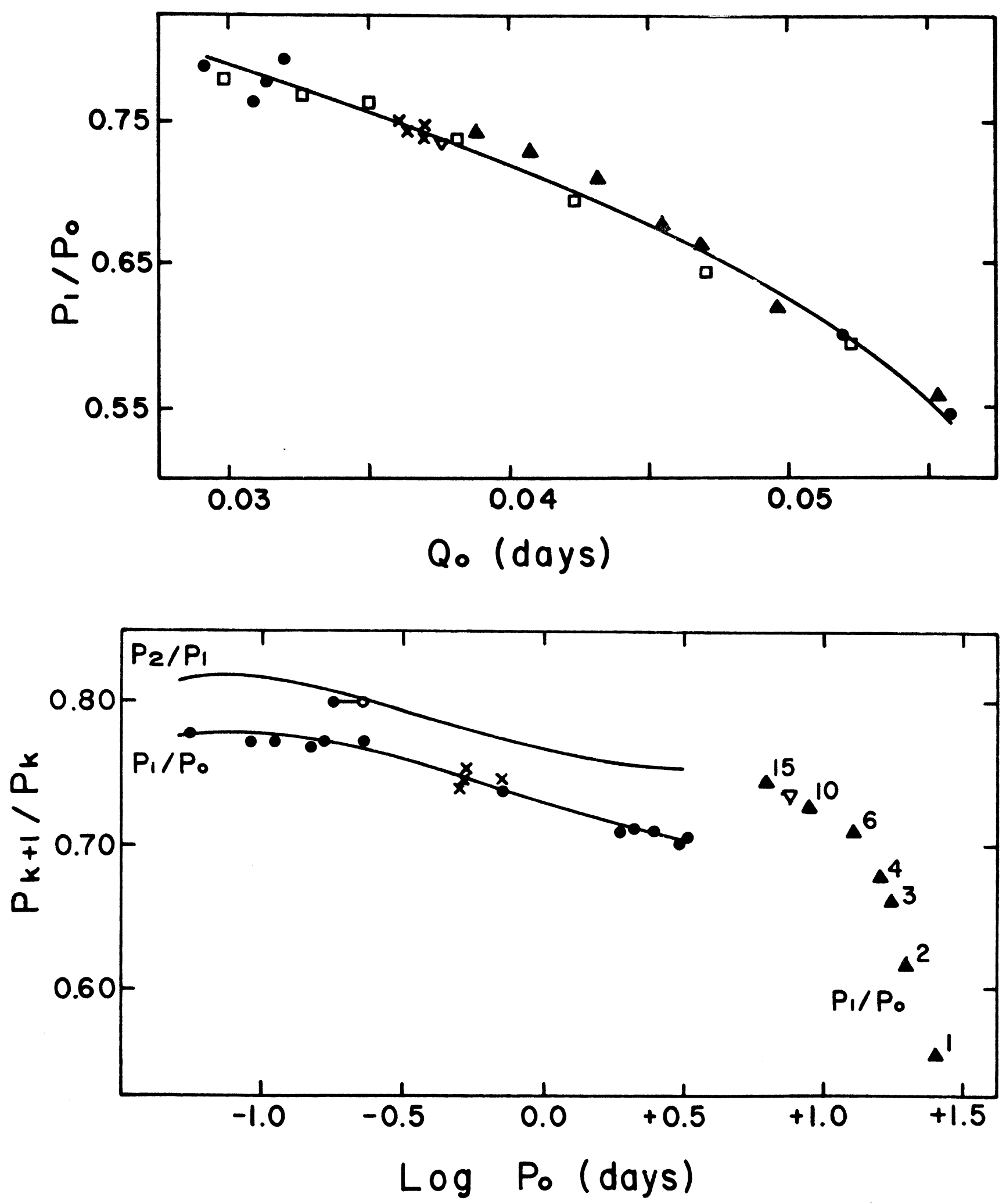